\title{
Daptomycin-resistant Staphylococcus pettenkoferi of human origin*
}

\author{
Vincenzo Savini ${ }^{1}$, Maja Kosecka², Ed Siegwart ${ }^{3}$, Roberta Marrollo', Ennio Polilli ${ }^{1}$, \\ Dalia Palmieri4 ${ }^{4}$ Paolo Fazii ${ }^{1}$, Edoardo Carretto ${ }^{5}$, Jacek Międzobrodzki² and Michal Bukowski6 ${ }^{\bowtie}$ \\ ${ }^{1}$ Clinical Microbiology and Virology, Spirito Santo Hospital, Pescara, Italy; ${ }^{2}$ Department of Microbiology, Faculty of Biochemistry, Biophysics and \\ Biotechnology, Jagiellonian University, Kraków, Poland; ${ }^{3 L G C ~ L t d, ~ N e w m a r k e t ~ R d, ~ F o r d h a m, ~ C a m b r i d g e s h i r e, ~ U n i t e d ~ K i n g d o m ; ~}{ }^{E}$ Epidemiological \\ Office, Spirito Santo Hospital, Pescara, Italy; ${ }^{5}$ Laboratory of Microbiology, IRCCS Arcispedale Santa Maria Nuova, Reggio Emilia, Italy; ${ }^{6}$ Department \\ of Analytical Biochemistry, Faculty of Biochemistry, Biophysics and Biotechnology, Jagiellonian University, Kraków, Poland
}

The importance of nosocomial infections caused by coagulase-negative staphylococci is constantly growing. The threat primarily affects immunocompromised patients, the elderly and neonates, particularly after invasive surgery. The problem is fundamentally exacerbated by expanding antibacterial drug resistance. A case report is presented of an 86-year-old patient who underwent a ruptured abdominal aortic aneurysm surgery and developed septicaemia upon surgical wound infection. The causal agent was likely a carbapenem-resistant Klebsiella pneumoniae, however, daptomycin-resistant Staphylococcus pettenkoferi was identified in blood cultures in the absence of daptomycin treatment. To the authors' knowledge, the case study presented is the first published episode of daptomycin-resistant S. pettenkoferi strain.

Key words: daptomycin resistance, coagulase-negative staphylococci, Staphylococcus pettenkoferi, Klebsiella pneumoniae

Received: 30 July, 2015; revised: 23 October, 2015; accepted: 16 December, 2015; available on-line: 07 January, 2016

\section{INTRODUCTION}

Staphylococci are common commensals that frequently colonise skin and mucous membranes of healthy people. Nonetheless, these bacteria often behave as opportunistic pathogens which can lead to life-threatening local and systemic infections (Rasigade \& Vandenesch, 2014). For historical reasons, clinical diagnosis is mainly focused on coagulase-positive staphylococci (CoPS), predominantly Staphylococcus aureus, which can lead to underestimation of coagulase-negative staphylococci (CoNS) pathogenicity (Morfin-Otero et al., 2012). However, there is an increasing number of reports suggesting that CoNS are also an important cause of infections in hospitalised patients (Wójkowska-Mach et al., 2014). This phenomenon may be exemplified by the case of Staphylococcus pettenkoferi. The latter is a coagulase-negative species forming smooth, slightly convex, glistening and opaque colonies (some isolates are yellow-orange pigmented), named in honor of Max von Pettenkofer (1818-1901), who was a pioneer in the field of hygiene and public health in the former kingdom of Bavaria. It was first described relatively recently (Trülzsch et al., 2002; Trülzsch et al., 2007) but has already been reported as an aetiological agent of wound (including those related to the diabetic foot) and bloodstream infections, osteomyelitis and bacteraemias in intensive care units (Krediet et al., 2004; Loïez et al.,
2007; Song et al., 2009; Mammina et al., 2011; Mihaila et al., 2012; Morfin-Otero et al., 2012; Park et al., 2015). The problem of CoNS infections in the clinical setting is made more severe as a result of antibiotic resistance spread (Davies \& Davies, 2010; Rasigade et al., 2012; Fluit et al., 2013; Mkrtchyan et al., 2013). Concerning staphylococci, the most alarming resistance phenotypes are those involving daptomycin, a drug which is one of only a few valid alternatives to treat diseases caused by methicillin- and vancomycin-resistant strains (Tótoli et al., 2015). In the case study presented here, we report what appears to be a unique description of daptomycin-resistant $S$. pettenkoferi human isolate collected from blood.

\section{CASE REPORT}

Background. An 86-year-old male patient was admitted in June 2014 to the Civic Hospital of Pescara, Italy, due to an apparent wound infection (cultures were not performed), which had developed after ruptured abdominal aortic aneurysm surgery. A central venous catheter (CVC) was placed into the right internal jugular vein in order to administer systemic antibiotics. Despite several antimicrobial treatments, including ciprofloxacin, metronidazole meropenem, piperacillin/tazobactam, clindamycin, colistin, tigecycline and fluconazole, that the patient received in the two-month-period from hospitalization, his body temperature always kept between $36.8^{\circ} \mathrm{C}$ and $39.3^{\circ} \mathrm{C}$, and no improvement in the wound aspect was observed. Nevertheless, the supervising clinicians did not request microbiological evaluation of the wound exudate, and instead the CVC was removed and sent for culture; it was found that its tip, particularly, grew a few carbapenem-resistant Klebsiella pneumoniae colonies (description of related methods is outside the scope of this paper). Concomitantly, blood samples were taken and sent to the Microbiology Laboratory, as described in detail below.

e-mail: m.bukowski@uj.edu.pl

*The results were presented at the 6th International Weigl Conference on Microbiology, Gdańsk, Poland (8-10 July, 2015).

Abbreviations: CoPS, coagulase-positive staphylococci; CoNS coagulase-negative staphylococci; CVC, central venous catheter; EUCAST, European Committee for Antibiotic Susceptibility Testing; CLSI, Clinical and Laboratory Standards Institute; MIC, minimum inhibitory concentration; MALDI ToF MS, matrix-assisted laser desorption/ionization time of flight mass spectrometry; HCCA, alphacyano-4-hydroxycinnamic acid; RFLP, restriction fragments length polymorphism; CA-MHB, cation adjusted Mueller Hinton broth; $\mathrm{PCR}$, polymerase chain reaction 
Despite treatment, the patient died due to septic shock 2 months after hospitalization.

Cultures. Two 4-mL blood samples were inoculated into two BacT/Alert bottles (bioMérieux, France, one for aerobes and one for anaerobes) and were scored as positive after 24 hour incubation. A drop from each bottle was inoculated onto agar-based media to cultivate both, aerobic and anaerobic organisms, if present, and incubated at $36 \pm 1^{\circ} \mathrm{C}$. Microscopic examination carried out at the same time revealed the presence of Grampositive cocci in the aerobe-dedicated bottle. Catalasepositive, coagulase-negative, Gram-positive cocci were documented after 24-hour incubation and conclusively observed to represent a pure culture after 48 hours.

Identification and antibiotic susceptibility testing with Vitek2. The Vitek2 GP card (bioMeriéux, France) identified the isolate as Staphylococcus capitis, with 99\% certainty (D'Azevedo et al., 2009). Also, the studied strain was found to be both, oxacillin- and daptomycinresistant, by using the Vitek2 AST632 card (bioMeriéux, France).

Search for the mec genes. Real-Time Polymerase Chain Reaction performed with Xpert $^{\mathbb{B}}$ MRSA Gen 3 (Cepheid, US) and targeting both, mecA and mec $\mathrm{C}$ genes, provided negative results, so the isolate was conclusively considered as non-methicillin/oxacillin-resistant, despite the above mentioned results.

Susceptibility testing by agar-based methods. An agar disc diffusion method based on EUCAST (European Committee for Antibiotic Susceptibility Testing) 2015 criteria (medium for testing, incubation temperature and incubation time were Mueller-Hinton agar, $35 \pm 1^{\circ} \mathrm{C}$ and $18 \pm 2 \mathrm{~h}$, respectively) was carried out with a $30 \mu \mathrm{g}$ cefoxitin disk and gave an inhibition zone diameter of 22 $\mathrm{mm}$; the test was repeated in another laboratory, providing an inhibition zone diameter result of $23 \mathrm{~mm}$; both were therefore in the resistant range according to CLSI 2015 (Clinical and Laboratory Standards Institute) and EUCAST 2015 guidelines. Also, determination of daptomycin and oxacillin MICs was performed using Liofilchem ${ }^{\circledR}$ MIC Test Strips and the values obtained turned out to be $\geq 1.5 \mathrm{mg} / \mathrm{mL}$ (Fig. 1) and $0.5 \mathrm{mg} / \mathrm{mL}$, respectively. Hence, as reported by CLSI 2015 for oxacillin MIC interpretive criteria, cefoxitin screening might overestimate CoNS resistance to betalactams in some cases. Finally, daptomycin appeared to be ineffective, in vitro, on the studied strain.

Bruker Daltronic MALDI ToF identification. To allow further analysis, the staphylococcal isolate was reidentified using a MALDI ToF Biotyper. Matrix-assisted laser desorption/ionization (MALDI) is a soft ionization technique used in mass spectrometry. The MALDI Biotyper (Bruker, UK) utilises MALDI technology to measure ribosomal proteins and create a mass spectrum or 'fingerprint' unique to individual species of bacteria or fungi resulting in a high throughput and reliable method for the classification and identification of microorganisms (Seng et al., 2009; Dubois et al., 2010; Haigh et al., 2013).

A small amount of biological material was applied directly on a MALDI target plate in a thin smear. The thin microbial film was overlaid with $0.7 \mu \mathrm{l}$ of a saturated alpha-cyano-4-hydroxycinnamic acid (HCCA) matrix solution and air dried. The matrix protects the sample from being destroyed by the direct laser beam and facilitates vaporization and ionization. Inside the MALDI-TOF mass spectrometer, a $60 \mathrm{~Hz}$ nitrogen laser is fired 240 times at each sample and mass spectra are acquired between 2000 and $20000 \mathrm{Da}$ automatically.

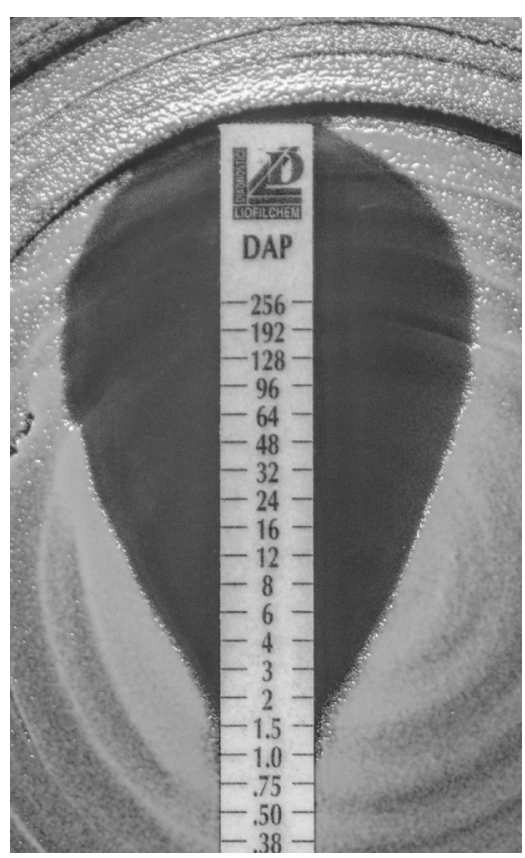

Figure 1. Determination of S. pettenkoferi s461 daptomycin resistance.

The minimal inhibitory concentration (MIC) is greater than $1 \mu \mathrm{g} /$ $\mathrm{ml}$, which allows for classification of the analysed strain as resistant, using the EUCAST and CLSI breakpoints.

The vaporized and ionised protein fragments are released into a tube (1-2 $\mathrm{m}$ in length), accelerated and allowed to drift towards a detector. Time-of-Flight (TOF) is proportional to the molecular weight of the fragment. After 240 laser shots, an accumulated spectrum of the sample will be produced.

The obtained mass spectrum will show a characteristic peak pattern known as the "fingerprint spectrum". "Pattern matching" the MS fingerprint spectrum with the reference library quickly identifies each microorganism. A matching score based on identified masses and the intensity correlation is generated and used for ranking of the results.

The tested strain gave an identification of Staphylococcus pettenkoferi and a matching score of 2.243 in the Bruker Daltronic realtime identification software with a strong match for strain Staphylococcus pettenkoferi KL906013_ BK14723 DT UKH.

Vitek MS identification. In contrast to the identification obtained with the aforementioned Bruker-Daltronic MALDI-TOF technology, the one provided by bioMérieux (Vitek MS) was not able to achieve any characterization for the studied organism, as the latter was on more than one occasion labelled as 'non-identified'. Of course, this was due to the absence of $S$. pettenkoferi profile in the related software library.

Genetic identification. RFLP (restriction fragments length polymorphism) patterns were obtained for gap (Yugueros et al., 2000; Sa et al., 2001) and saoC genes (Bukowski et al., 2015) according to the methodology described by their authors in the referenced literature. For the absence of a reference strain in the widely accessible reference collections, the patterns were compared with in silico ones generated for aforementioned genes, whose sequences were obtained from the whole genome shotgun sequence of $S$. pettenkoferi VCU012 (GenBank accession: AGUA01000000), the only genomic sequence available for this species. The in silico patterns were obtained using CLC Main Workbench software (CLC bio, Qiagen) 


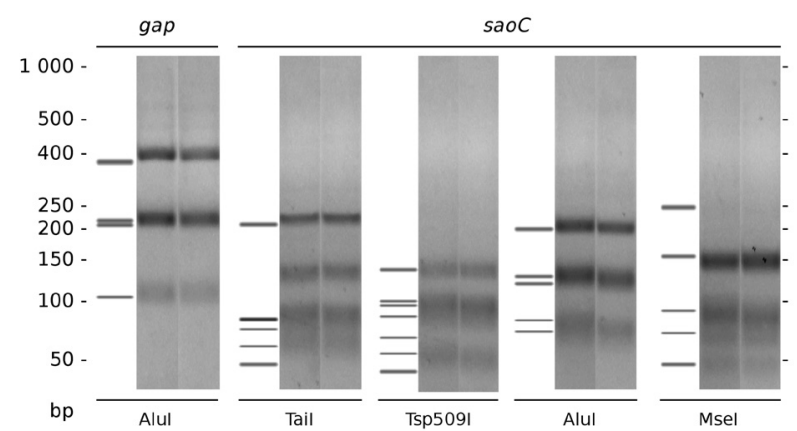

Figure 2. RFLP patterns for gap and saoC genes.

The patterns were obtained for the analysed Staphylococcus spp. strain in two replicas. First of each three lanes is an in silico pattern for the reference strain S. pettenkoferi VCU012. Below, restriction enzymes, corresponding to the patterns, are listed.

and compared to the in vitro ones using GelCompar II (Applied Biosystems). The gap patterns were identical between the reference and analysed strains (Fig. 2). For the saoC gene, 17 of 22 reference bands ideally corresponded to the obtained patterns, and for one of four different restriction enzymes, namely AluI, the pattern was identical to the reference one (Fig. 2). Importantly, the obtained patterns were unique when compared to those obtained for the other 26 staphylococci species (Bukowski et al., 2015). Polymorphisms for the saoC gene suggest a distant phylogenetic relation with the reference strain. The results were validated by $16 \mathrm{~S} r R N A$ and $r p o \mathrm{~B}$ sequencing (Drancourt \& Raoult, 2002; Becker et al., 2004). For the former, the obtained sequence (GenBank accession: KT199298) was the most similar and 99\% identical to the one for S. pettenkoferi B3117. The latter (GenBank accession: KT199299) was identical to the one for S. pettenkoferi CCM 7495. The results obtained using genetic methods clearly confirm the identity of the analysed strain as $S$. pettenkoferi, which was given a signature s461 (BioSample accession: SAMN03784716).

Broth dilution MIC Testing. A CLSI broth dilution method, following CLSI M100-S23 (2013) and CLSI M07-A9 (2012) guidelines was used to quantitatively determine the in vitro activity of daptomycin against the bacterial isolate. A microdilution broth method in which a sterile microtiter tray was inoculated with an antimicrobial dilution range was used, where the antimicrobial agent and a suspension of the tested organism were added together using an autoinoculator. After incubation, the MIC can be determined by examination of the plate for visible growth.

The antimicrobial potency for daptomycin was calculated using the purity value supplied, with corrections made for water content and the inactive fraction of salt preparations. All calculations were carried out according to the information supplied on the certificates of analy- sis. Stock solutions of antimicrobial agents and the tested substance were made using a specific mass (as stated on the manufacturer's certificate of analysis), calculated using the potency, required volume and stock concentration. A three step dilution scheme, as stated in CLSI M100-S23 (2013), was used to prepare the antimicrobial concentration range.

Once all required concentrations had been diluted at twice the required final concentration, they were loaded in $2.2 \mathrm{~mL}$ aliquots into polypropylene 96 well, deep well blocks. Deep well blocks were then used to dispense 50 $\mu \mathrm{L}$ aliquots into 96 well microtiter plates using an automated liquid handling system. These plates were then stored frozen at nominal $-80^{\circ} \mathrm{C}$, and thawed prior to testing.

As stated in CLSI and EUCAST guidelines, calcium ion concentration within the tested media is critical to ensure reproducibility and reliability of the results in case of daptomycin testing. The calcium ion content of the prepared broth microdilution plates (once inoculated) was calculated using an Olympus AU640 analyser. Calcium $\left(\mathrm{Ca}^{2+}\right)$ content in tested plates was $49.6 \mathrm{mg} / \mathrm{L}$ and falls within acceptable limits stated in the EUCAST guidelines.

Test plates were prepared using an inoculum solution containing $2-8 \times 10^{5} \mathrm{CFU} / \mathrm{mL}$ of the tested organism (colony forming units $/ \mathrm{mL}$ ). This solution was made firstly by selecting 3-5 colonies from an overnight subculture plate, and using that to prepare a $0.5 \mathrm{McF}$ arland solution in $5 \mathrm{~mL}$ of cation adjusted Mueller Hinton broth (CA-MHB), which contained approximately $10^{8} \mathrm{CFU} /$ $\mathrm{mL}$ of the tested culture. $80 \mu \mathrm{L}$ of this solution was added to $11 \mathrm{~mL}$ CA-MHB to form an inoculum solution of approximately $2-8 \times 10^{5} \mathrm{CFU} / \mathrm{mL}$. This was then added in $50 \mu \mathrm{L}$ aliquots to the pre-prepared 96 well microtiter plates using an auto inoculating machine and incubated as per conditions stated in the CLSI guidelines.

Following incubation, MIC results were read by macroscopic evaluation, recorded on controlled paperwork and checked by a second scientist. The MIC result was $2 \mu \mathrm{g} / \mathrm{ml}$, which allowed for classification of the analysed strain as resistant using EUCAST and CLSI breakpoints (CLSI and EUCAST: resistant breakpoint $>1 \mu \mathrm{g} / \mathrm{mL}$ ).

MIC results were confirmed using E-test strips following manufacturer's instructions and using Mueller Hinton agar (Fig. 1).

Putative mechanism of daptomycin resistance. Using the primers mprF-F: 5'-TAAT'TAGACATTAAGAATTGAGGATGAC-3'; and mprF-R: 5'-ACACTTAACGTCATAACCTCT'TC-3'; a PCR product of $m p r F$ for the analysed $S$. pettenkoferi strain was obtained. Because of its length, the product was subjected to Sanger sequencing using two additional primers besides those aforementioned: mprF-in1-F: 5'-T'TATTGTACCATCATT'TCGAGTCTTG-3'; mprF-in2-F: 5'-TATGCATCTTATAT'TCACTGCTATG-3'. The $m p r F$ gene codes for phosphatidylglycerol lysyltransferase, which is

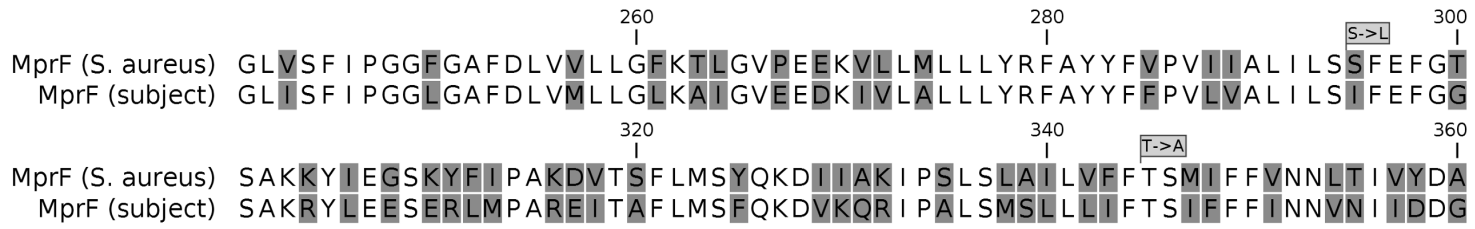

Figure 3. MprF sequence similarity between S. aureus and S. pettenkoferi.

The picture presents a fragment of an alignment between MprF sequence of S. aureus and the analysed S. pettenkoferi strain (subject). The varying amino acids are highlighted. The sites of the mutation mediating daptomycin resistance in S. aureus are marked above the sequence (S295L and T345A). Noticeably in the position 295 of the MprF sequence, the analysed S. pettenkoferi strain possesses isoleucine (I). 


\section{0 760 780 MprF (reference) NLMPTYYEDTLSVNLLIRWGSETDLPLMDVLYLN I LLWAQEQGYRHFNMGMATLSNVGQAS MprF (subject) NLMPTYYEDTLSVDL I RWGSETDLPLMDVLYLN I LLWAQKQGYRHFNMGMATLSNVGQAS}

Figure 4. MprF sequence diversity within S. pettenkoferi.

The picture presents a fragment of an alignment between MprF sequence of S. pettenkoferi VCU012 (reference) and of the analysed S. pettenkoferi strain (subject). Two mutations leading to a charge change of two amino acid residues are highlighted (N734D, E760K).

responsible for phosphatidiloglicerol lysinilation. Missense mutations within $m p r F$ may mediate membranecharge-dependent resistance against daptomycin (Yang et al., 2013; Bayer et al., 2015; Yamaguchi et al., 2015). As it was demonstrated for $S$. aureus, two missense mutation in the mprF gene may lead to the resistant phenotype (Yang et al., 2013). These mutations lead to serine (Ser, S) to leucine (Leu, L) change in the position 295 and threonine (Thr, T) to alanine (Ala, A) change in the position 345. However, the MprF sequence for S. pettenkoferi differs significantly from that for $S$. aureus (Fig. 3); the one obtained for the analysed strain (GenBank accession: KT199300) has isoleucine (Ile, I) in the position corresponding to the position 345 for $S$. aureus. The same difference is observed for $S$. pettenkoferi VCU012, the only strain of this species with known $m p r F$ sequence (GenBank accession: AGUA01000000). Unfortunately, its susceptibility to daptomycin is unknown.

Nonetheless, when the S. pettenkoferi VCU012 MprF sequence is compared to the one for the analysed strain, two additional changes were identified (Fig. 4). These are asparagine (Asn, N) to aspartic acid (Asp, D) change in the position 734 and glutamic acid (Glu, E) to lysine (Lys, K) in the position 760 . Such changes of the charged residues may alter MprF activity and may be involved in the daptomycin resistance. Such a phenomenon, related not only to daptomycin, may be an effect of cumulative missense mutations in $m p r F$, completely different from the two mentioned in the beginning of this section, and other genes, which leads to membrane structure changes (Young et al., 2012). Undoubtedly, daptomycin resistance of the analysed $S$. pettenkoferi strain remains an attractive issue to be explored in future research.

\section{DISCUSSION AND CONCLUSIONS}

The exitus of the case presented was presumably due to an undetected carbapenem-resistant $K$. pneumoniae infection, while the bloodstream-related S. pettenkoferi isolate was a putative skin saprophyte accidentally introduced into the BacT/Alert bottle, possibly by virtue of inadequate preparation of the cutaneous venipuncture site.

Despite the fact that most likely the isolate represented a harmless contaminant, it was intriguing to observe that it displayed a daptomycin-resistant phenotype. As daptomycin resistance was observed in a methicillin/ oxacillin-susceptible strain, it has been of further interest to note that these two resistance traits may not be necessarily related to each other or be concomitant. Moreover, such a finding was even more intriguing based on the fact that the patient had no noted prior daptomycin treatment. We could therefore speculate that the strain acquired the resistance trait elsewhere, before it reached the patient's skin and was transferred to the venipuncuture site perhaps by means of caretakers' hands, and finally reached the bottle for blood collection due to the phlebotomy procedure. This is, however, no more than a hypothesis.
Another theory is that the $S$. pettenkoferi strain developed resistance whilst the patient was being treated for the $K$. pneumoniae infection, as antimicrobial levels reaching what is likely to have been a skin flora organism would most likely be sub-inhibitory, encouraging resistance development. Also, as the $K$. pneumoniae infection was notably resistant, a range of antibiotics may have been used to treat the patient (including prophylactic antibiotics for surgical preparation), increasing the chance of exposure of the flora to a wide range of compounds, and therefore increasing resistance development potential throughout the flora.

We believe a leading message may be taken home from the aforementioned observations; such a message focuses on CoNS behaviour as potential reservoirs for antibiotic resistance traits, independently from their commensal or pathogenic role. It is therefore important from both, a medical and epidemiological perspective, that clinical microbiologists who encounter CoNS isolates daily in the hospital practice, do not aprioristically dismiss them as innocent bystanders and that they investigate the resistant traits each strain may display. The importance of correct and complete identification of microbiological samples is also an issue of note. As with the case study presented here, a number of differing identification methods has been used and some either failed to produce a result or provided an incorrect result.

Finally, daptomycin resistance in CoNS is still an open field of research. It is then crucial that phenotypical observations should be supported by genome-based studies on molecular mechanisms behind a clinically evident lack of in vitro drug activity, especially when the latest compounds are involved, such as glycopeptides, linezolid, or daptomycin, that represent important weapons to face potentially life-threatening staphylococcal diseases throughout the hospital wards.

\section{Acknowledgements}

This research was partially financed by funds granted by the National Science Centre (NCN, Poland) on the basis of the decision no. DEC-2011/01/N/NZ1/01167 (to MB). Faculty of Biochemistry, Biophysics and Biotechnology of Jagiellonian University is a partner of the Leading National Research Center (KNOW) supported by the Ministry of Science and Higher Education, Warsaw, Poland.

\section{REFERENCES}

Bayer AS, Mishra NN, Chen L, Kreiswirth BN, Rubio A, Yang S-J (2015) Frequency and distribution of single nucleotide polymorphisms within $m p r F$ in methicillin-resistant Stapbylococcus aureus (MRSA) Clinical Isolates: role in cross-resistance between daptomycin and host defense antimicrobial peptides. Antimicrob Agents and Chemother [Epub ahead of print] http://dx.doi.org/10.1128/ AAC.00970-15

Becker K, Harmsen D, Mellmann A, Meier C, Schumann P, Peters $G$, von Eiff C (2004) Development and evaluation of a qualitycontrolled ribosomal sequence database for $16 \mathrm{~S}$ ribosomal DNAbased identification of Staphylococcus species. I Clin Microbiolol 42): 4988-4995.

Bukowski M, Polakowska K, Ilczyszyn WM, Sitarska A, Nytko K, Kosecka M, Miedzobrodzki J, Dubin A, Wladyka B (2015) Species de- 
termination within Staphylococcus genus by extended PCR-restriction fragment length polymorphism of saoC gene. FEMS Microbiol Lett 362: 1-11. http://dx.doi.org/10.1093/femsle/fnu007.

Cockerill FR, Wikler MA, Alder J, Dudley MN, Eliopoulos GM, Ferraro MJ, Hardy DJ, Hecht DW, Hindler JA, Patel JB, Powell M, Swenson JM, Jr. RBT, Traczewski MM, Turnidge JD, Weinstein MP, Zimmer BL (2013) Performance standards for antimicrobial susceptibility testing; twenty-second informational supplement, Clinical and Laboratory Standards Institute.

Cockerill FR, Wikler MA, Alder J, Dudley MN, Eliopoulos GM, Ferraro MJ, Hardy DJ, Hecht DW, Hindler JA, Patel JB, Powell M, Swenson JM, Richard B. Thomson J, Traczewski MM, Turnidge JD, Weinstein MP, Zimmer BL (2012) Methods for dilution antimicrobial susceptibility tests for bacteria that grow aerobically; approved standards 9th edn, Clinical and Laboratory Standards Institute.

Davies J, Davies D (2010) Origins and evolution of antibiotic resistance. Microbiol Mol Biol Rev 74: 417-433. http://dx.doi.org/10.1128/ MMBR.00016-10.

Drancourt M, Raoult D (2002) rpoB gene sequence-based identification of Staphylococcus species. J Clin Microbiol 40: 1333-1338.

Dubois D, Leyssene D, Chacornac JP, Kostrzewa M, Schmit PO, Talon R, Bonnet R, Delmas J (2010) Identification of a variety of Staphylococcus species by matrix-assisted laser desorption ionizationtime of flight mass spectrometry. J Clin Microbiol 48: 941-945. http://dx.doi.org/10.1128/JCM.00413-09.

D’Azevedo PA, Siquiera I, Gugel J, Antunes AL, Secchi C, Pasternak J, Martino MD (2009) Evaluation of the automated system Vitek2 for identification and antimicrobial susceptibility testing of Brazilian Gram-positive cocci strains. Braz J Infect Dis 13: 107-110.

Fluit AC, Carpaij N, Majoor EAM, Bonten MJM, Willems RJL (2013) Shared reservoir of ccr $\mathrm{B}$ gene sequences between coagulase-negative staphylococci and methicillin-resistant Staphylococcus aureus. I Antimicrob Chemother 68: 1707-1713. http://dx.doi.org/10.1093/jac/dkt121.

Haigh JD, Green IM, Ball D, Eydmann M1, Millar M1, Wilks M (2013) Rapid identification of bacteria from bioMérieux BacT/ ALERT blood culture bottles by MALDI-TOF MS. Br J Biomed Sci 70: 149-155.

Krediet TG, Mascini EM, van Rooij E, Vlooswijk J, Paauw A, Gerards LJ, Fleer A (2004) Molecular epidemiology of coagulase-negative staphylococci causing sepsis in a neonatal intensive care unit over an 11-year period. J Clin Microbiol 42: 992-995.

Loïz C, Wallet F, Pischedda P, Renaux E, Senneville E, Mehdi N, Courcol RJ (2007) First case of osteomyelitis caused by Staphylococcus pettenkoferi. J Clin Microbiol 45: 1069-1071.

Mammina C, Bonura C, Verde MS, Fasciana T, Palma DM (2011) A fatal bloodstream infection by Staphylococcus pettenkoferi in an intensive care unit patient. Case Rep Crit Care 2011: 612732. http:// dx.doi.org/10.1155/2011/612732.

Mihaila L, Defrance G, Levesque E, Ichai P, Garnier F, Derouin V, Decousser JW, Doucet-Populaire F, Bourgeois-Nicolaos N (2012) A dual outbreak of bloodstream infections with linezolid-resistant Staphylococcus epidermidis and Staphylococcus pettenkoferi in a liver Intensive Care Unit. Int J Antimicrob Agents 40: 472-474. http://dx.doi. org/10.1016/j.ijantimicag.2012.06.014

Mkrtchyan H V, Russell CA, Wang N, Cutler RR (2013) Could public restrooms be an environment for bacterial resistomes? PloS One 8: e54223. http://dx.doi.org/10.1371/journal.pone.0054223.

Morfin-Otero R, Martínez-Vázquez MA, López D, Rodríguez-Noriega E, Garza-González E (2012) Isolation of rare coagulase-negative isolates in immunocompromised patients: Staphylococcus gallinarum, Staphylococcus pettenkoferi and Staphylococcus pasteuri. Ann Clin Lab Sci 42: 182-185.

Park S, Chung H-S, Lee M (2015) Clinical and microbiological characteristics of six Staphylococcus pettenkoferi isolates from blood sam- ples. Ann of Lab Med 35: 250-253. http://dx.doi.org/10.3343/ alm.2015.35.2.250

Rasigade J-P, Raulin O, Picaud J-C, Tellini C, Bes M, Grando J, Ben Saïd M, Claris O, Etienne J, Tigaud S, Laurent F (2012) Methicillinresistant Staphylococcus capitis with reduced vancomycin susceptibility causes late-onset sepsis in intensive care neonates. PLoS One 7: e31548. http://dx.doi.org/10.1371/journal.pone.0031548.

Rasigade JP, Vandenesch F (2014) Staphylococcus aureus: A pathogen with still unresolved issues. Infect Genet Evol 21: 510-514. http://dx.doi. org/10.1016/j.meegid.2013.08.018.

Sa A, Yugueros J, Temprano A, Luengo A, Animal DDS, Sánchez M, Luengo JM, Naharro G (2001) Identification of Staphylococcus spp. by PCR-restriction fragment length polymorphism of gap gene. $J$ Clin Microbiol 39: 3693-3695.

Seng P, Drancourt M, Gouriet F, La Scola B, Fournier PE, Rolain JM, Raoult D (2009) Ongoing revolution in bacteriology: routine identification of bacteria by matrix-assisted laser desorption ionization time-of-flight mass spectrometry. Clin Infect Dis 49: 543-551. http:// dx.doi.org/10.1086/600885.

Song SH, Park JS, Kwon HR, Kim SH, Kim H Bin, Chang HE, Park KU, Song J, Kim EC (2009) Human bloodstream infection caused by Staphylococcus pettenkoferi. J Med Microbiol 58: 270-272. http:// dx.doi.org/10.1099/jmm.0.004697-0.

Tótoli EG, Garg S,Nunes Salgado HR (2015) Daptomycin: physicochemical, analytical and pharmacological properties. Ther Drug Monit [Epub ahead of print].

Trülzsch K, Grabein B, Schumann P, Mellmann A, Antonenka U, Heesemann J, Becker K (2007) Staphylococcus pettenkofferi sp. nov., a novel coagulase-negative staphylococcal species isolated from human clinical specimens. Int J Syst Evol Microbiol 57: 1543-1548.

Trülzsch K, Rinder H, Trcek J, Bader L, Wilhelm U, Heesemann J (2002) Staphylococcus pettenkoferi a novel staphylococcal species isolated from clinical specimens. Int J Syst Evol Microbiol 43: 175-182.

Wójkowska-Mach J, Gulczyńska E, Nowiczewski M, Borszewska-Kornacka M, Domańska J, Merritt TA, Helwich E, Kordek A, Pawlik D, Gadzinowski J, Szczapa J, Adamski P, Sulik M, Klamka J, Brzychczy-Włoch M, Heczko PB (2014) Late-onset bloodstream infections of very-low-birth-weight infants: data from the Polish Neonatology Surveillance Network in 2009-2011. BMC Infect Dis 18: 339. http://dx.doi.org/10.1186/1471-2334-14-339.

Yamaguchi T, Suzuki S, Okamura S, Miura Y, Tsukimori A, Nakamura I, Ito N, Masuya A, Shiina T, Matsumoto T (2015) Evolution and single-nucleotide polymorphisms in methicillin-resistant Staphylococcus aureus strains with reduced susceptibility to vancomycin and daptomycin, based on determination of the complete genome. Antimicrob Agents and Chemother 59: 3585-3587. http://dx.doi.org/10.1128/ AAC.05159-14.

Yang S-J, Mishra NN, Rubio A, Bayer AS (2013) Causal role of single nucleotide polymorphisms within the mprF gene of Staphylococcus aureus in daptomycin resistance. Antimicrob Agents and Chemother 57: 5658-5664. http://dx.doi.org/10.1128/AAC.01184-13.

Young BC, Golubchik T, Batty EM, Fung R, Larner-Svensson H, Votintseva AA, Miller RR, Godwin H, Knox K, Everitt RG, Iqbal Z, Rimmer AJ, Cule M, Ip CLC, Didelot X, Harding RM, Donnelly P, Peto TE, Crook DW et al. (2012) Evolutionary dynamics of Staphylococcus aureus during progression from carriage to disease. Proc Natl Acad Sci USA 109: 4550-4555. http://dx.doi.org/10.1073/ pnas.1113219109.

Yugueros J, Temprano A, Berzal B, Sánchez M, Hernanz C, Luengo JM, Naharro G (2000) Glyceraldehyde-3-phosphate dehydrogenaseencoding gene as a useful taxonomic tool for Staphylococcus spp. J Clin Microbiol 38: 4351-4355. 